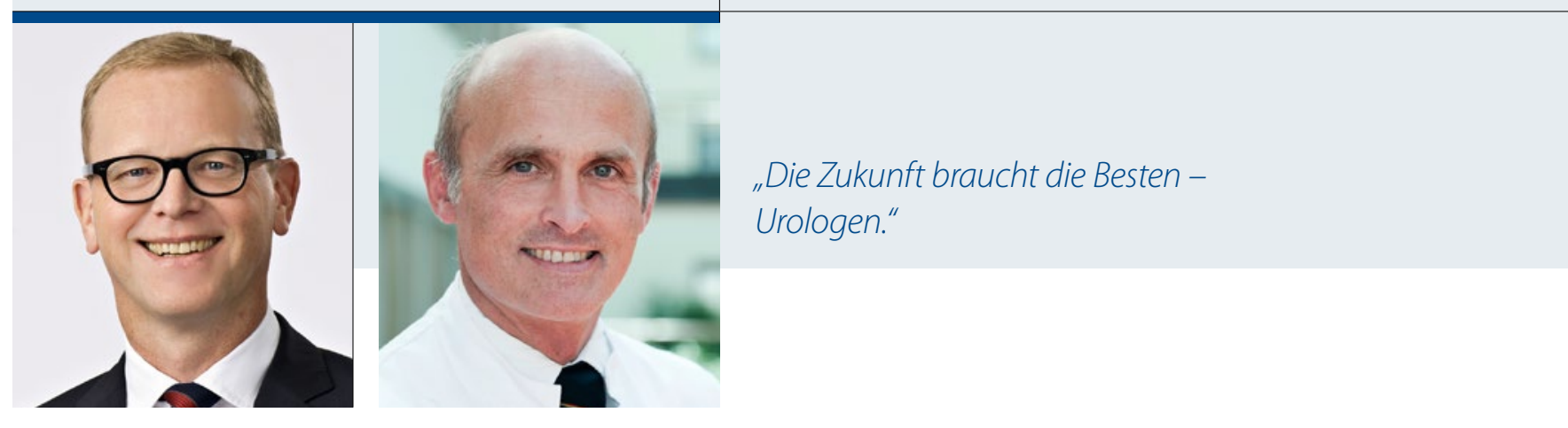

Prof. Dr. med. Elmar W. Gerharz Prof. Dr. med. Andreas J. Gross

Urologie an der Paulskirche, Klinik für Urologie, Asklepios Klinik

UROGATE, Frankfurt am Main Barmbeck, Hamburg

\title{
Que sera - Was wird sein?
}

D ie geläufigste Version des Gassenhauers ist wohl die gutgelaunt dialogische von Doris Day, in der das All-American-Girl am Ende jeder Strophe gelassen altklug zu dem Schluss kommt „The future's not ours to see“. Auch wir Urologen können nicht in die Zukunft sehen. Was aber keinesfalls heißt, dass wir schicksalsergeben alles hinnehmen müssen, was wir uns selbst oder andere uns einzubrocken drohen. Kierkegaard schrieb einst, dass das Leben nur rückwärts verstanden werden kann, aber vorwärts gelebt werden muss. Wir gehen weiter und sagen, dass es vorwärts gestaltet werden muss, mit regelmäßigen systematischen und kritischen Bestandsaufnahmen und klar definierten strategischen Zielen. Das erfordert ein hohes $\mathrm{Maß}$ an Professionalität, Selbstdisziplin und eine intelligente Öffentlichkeitsarbeit. Während Präsidenten von Fachgesellschaften mit ihren befristeten Akzenten und Agenden kommen und gehen, haben die Generalsekretäre zweifellos das größere politische Gewicht. Bei dem anstehenden Personalwechsel sollten wir die Gelegenheit nutzen, nochmal die „Fahrkarten“ zu kontrollieren.

Wir haben entschieden, die diesjährige Kongressausgabe FUTURO-NEWS zu nennen und unsere Blicke durch ein Teleskop (s. Titelbild) zu werfen. Hierzu gehört ein Nachspiel zum kinderurologischen Schwerpunktheft: Dazu finden Sie einen Leserbrief unserer kinderchirurgischen Freunde, unsere kurze Replik, eine Stellungnahme von Kurt Miller und eine Aufforderung zu „Kooperation statt Konfrontation “von Wolfgang Rösch. Besonders hat uns gefreut, dass die Kinderchirurgen unseren Begriff „Uro-Think“ aufgegriffen haben, soll dieser Terminus in dieser Ausgabe doch eine große Rolle spielen. Mit der Zukunft der Urologie beschäftigen sich nämlich eine ganze Reihe ausgewiesener Uro-Denker, angeführt von dem unkonventionellen UroWeisen Peter Alken, der "Rausch und Reigen“ in der Urologie aus der Sicht des tagesgeschäftsfernen Un- ruheständlers erörtert und seine Analyse zuversichtlich schließt: „Wir sind die Besten - Urologen“. Mit Christopher Woodhouse schreibt ein zweiter Emeritus und erstmals ein englischer Erbadeliger - der sechste Lord Terrington - in URO-NEWS. Sein Beitrag zur Zukunft der rekonstruktiven Urologie ist elektronisch auch im englischsprachigen Original verfügbar. In seinem Impulsreferat legt der noch mitten im operativen Geschäft stehende Axel Heidenreich seine domstädtische Sicht auf die urologische Zukunft dar und fordert ,neue Strukturen und Wege“ aufzugreifen und zu nutzen „,bevor wir überholt werden".

Am Beispiel unseres Schicksalstumors beschreiben der frisch gekürte Präsident der Deutschen Krebsgesellschaft Peter Albers moderne ProstatakarzinomFrüherkennung als Next Generation Screening und der Martini-Philosoph Thorsten Schlomm, wie Internet, Big Data, Swarm Intelligence und Genomforschung unseren klinischen Alltag maßgeblich verändern werden. Ergänzt werden diese Weissagungen durch einen neuen Ansatz in der klinischen Entscheidungsfindung, beschrieben von Johannes $\mathrm{Hu}$ ber, und zwei Artikel zu dem wohl dramatischsten Aspekt unserer Zukunft, der Geriatrisierung der Medizin. Während Gerald Kolb eine Antwort auf die Frage „Quo vadis, Geriatrie?" versucht, informiert uns Bernd Kleine-Gunk, Präsident der German Society of Anti-Aging Medicine, wie man dem Problem durch ,ewige Jugend“ aus dem Weg gehen kann. Da die anspruchsvolle CME ein integraler Teil unserer Zukunft ist, runden die Hofer Kollegen mit ihren umfangreichen Erfahrungen in der modernen Harnröhrenchirurgie den URO-NEWS-Herbst ab.

Wir bedanken uns bei allen, die sich hier mit ihren Gedanken exponieren. Die Zukunft braucht die Besten - Urologen.

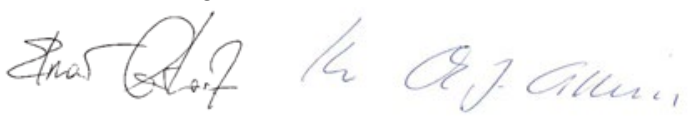

